\title{
A Study on New q-Integral Inequalities
}

\author{
Waad T. Sulaiman \\ Department of Computer Engineering, College of Engineering, University of Mosul, Mosul, Iraq \\ E-mail: waadsulaiman@hotmail.com \\ Received February 12, 2011; revised March 4, 2011; accepted March 7, 2011
}

\begin{abstract}
A q-analog, also called a q-extension or q-generalization is a mathematical expression parameterized by a quantity q that generalized a known expression and reduces to the known expression in the limit $q \rightarrow 1^{-}$. There are q-analogs for the fractional, binomial coefficient, derivative, Integral, Fibonacci numbers and so on. In this paper, we give several results, some of them are new and others are generalizations of the main results of [1]. As well as we give a generalization to the key lemma ([2], Lemma 1.3).
\end{abstract}

Keywords: q-Integral Inequlalities, Integral Inequalities

\section{Introduction}

For $0<q<1$, the q-analog of the derivative of the function $f(x)$, denoted by $D_{q} f(x)$ is defined (see [5]) by

$$
D_{q} f(x)=\frac{f(x)-f(q x)}{(1-q) x}, \quad x \neq 0
$$

If $f^{\prime}(0)$ exists, then $D_{q} f(0)=f^{\prime}(0)$. The q-derivative reduces to the usual derivative as $q \rightarrow 1$.

The q-analog of integration may be given (see [6]) by

$$
\int_{0}^{1} f(x) \mathrm{d}_{q} x=(1-q) \sum_{k=0}^{\infty} f\left(q^{k}\right) q^{k},
$$

which reduces to $\int_{0}^{1} f(x) \mathrm{d} x$ as $q \rightarrow 1$.

The q-Jackson integral from 0 to $a \in \mathfrak{R}$, for a more general case, can be defined (see [2,3]) by

$$
\int_{0}^{a} f(x) \mathrm{d}_{q} x=a(1-q) \sum_{k=0}^{\infty} f\left(a q^{k}\right) q^{k}
$$

provided the sum converges absolutely. The q-Jackson integral on a general interval may be defined (see $[2,3]$ ) by

$$
\int_{a}^{b} f(x) \mathrm{d}_{q} x=\int_{0}^{b} f(x) \mathrm{d}_{q} x-\int_{0}^{a} f(x) \mathrm{d}_{q} x .
$$

The q-Jackson integral and q-derivative are related by the "fundamental theorem of quantum calculus" which can be restated ([3, p.73]) as follows: If $F$ is an anti q-derivative of the function $f$, that is $D_{q} F=f$, continuous at $x=a$, then

$$
\int_{a}^{b} f(x) \mathrm{d}_{q} x=F(b)-F(a) .
$$

For any function $f$, we have

$$
D_{q}\left(\int_{a}^{x} f(t) \mathrm{d}_{q} t\right)=f(x) .
$$

It is not difficult to check that the q-analog of Leibniz's rule is

$$
D_{q}(f(x) g(x))=f(x) D_{q} g(x)+g(q x) D_{q} f(x) .
$$

For $b>0$ and $a=b q^{n}$ with $n \in \mathrm{N}$, we denote

$$
[a, b]_{q}=\left\{b q^{k}: 0 \leq k \leq n\right\} \text { and }(a, b]_{q}=\left[a q^{-1}, b\right]_{q} \text {. }
$$

Some applications of q-integrals:

$$
\int_{0}^{1} x \mathrm{~d}_{x} x=\frac{1}{1-q}, \int_{0}^{1} x^{n} \mathrm{~d}_{q} x=\frac{q-1}{q^{n}-1}, \int_{0}^{1} \ln x \mathrm{~d}_{q} x=\frac{q \ln q}{1-q},
$$

$\int_{0}^{\infty} \frac{x^{a-1}}{1-x} \mathrm{~d}_{q} x=\frac{\left(\Gamma_{q}\left(\frac{1}{2}\right)\right)^{2}}{\sigma_{q}(a)}$, where $\sigma_{q}(a)$ is a doubly periodic function. If $q=1$, the integral reduces to $\int_{0}^{\infty} \frac{x^{a-1}}{1-x} \mathrm{~d} x=\frac{\pi}{\sin (\pi a)}$.

In [4], the authors proved the following results:

Theorem 1.1. If $f(x)$ is a non-negative and increasing function on $[a, b]_{q}$ and satisfies

$$
(\alpha-1) f^{\alpha-2}(q x) D_{q} f(x) \geq \beta(\beta-1) f^{\beta-1}(x)(x-a)^{\beta-2}
$$


for $\alpha \geq 1$ and $\beta \geq 1$, then

$$
\int_{a}^{b} f^{\alpha}(x) \mathrm{d}_{q} x \geq\left(\int_{a}^{b} f(x) \mathrm{d}_{q} x\right)^{\beta} .
$$

Theorem 1.2. If $f(x)$ is a non-negative and increasing function on $\left[b q^{n+m}, b\right]_{q}$ for $m, n \in \mathrm{N}$ and satisfies $(\alpha-1) D_{q} f(x) \geq \beta(\beta-1) f^{\beta-\alpha+1}\left(q^{m} x\right)(x-a)^{\beta-2}$

on $[a, b]_{q}$ and for $\alpha, \beta \geq 1$, then

$$
\int_{a}^{b} f^{\alpha}(x) \mathrm{d}_{q} x \geq\left(\int_{a}^{b} f\left(q^{m} x\right) \mathrm{d}_{q} x\right)^{\beta} .
$$

Theorem 1.3. If $f(x)$ is a non-negative function on $[0, b]_{q}$ and satisfies

$$
\int_{x}^{b} f^{\beta}(t) \mathrm{d}_{q} t \geq \int_{x}^{b} t^{\beta} \mathrm{d}_{q} t
$$

for $x \in[0, b]_{q}$ and $\beta>0$, then the inequality

$$
\int_{0}^{b} f^{\alpha+\beta}(t) \mathrm{d}_{q} t \geq \int_{0}^{b} t^{\alpha} f^{\beta}(t) \mathrm{d}_{q} t
$$

holds for all positive numbers $\alpha$ and $\beta$.

In the coming section, we start with Lemma 2.1, which represent a generalization of ([1], Lemma 1.3). Theorems 2.3 and 2.4 are generalizations of Theorems 1.1 and 1.2 respectively, while Theorem 2.5 gives a generalization for more than one direction to Theorem 1.3. Other new results are also given.

\section{Results}

Lemma 2.1. Let $f(x), f^{\prime}(x), g(x)$ be nonnegative, nondecreasing functions defined on $[a, b]_{q}$, and let $p \geq 1$. Then

$$
\begin{aligned}
& p f^{p-1}(g(q x)) f^{\prime}(g(q x)) D_{q} g(x) \leq D_{q} f^{p}(g(x)) \\
& \leq p f^{p-1}(g(x)) f^{\prime}(g(x)) D_{q} g(x) .
\end{aligned}
$$

Proof. We have

$$
\begin{aligned}
& D_{q} f^{p}(g(x))=\frac{f^{p}(g(x))-f^{p}(g(q x))}{(1-q) x} \\
& =\frac{p}{(1-q) x} \int_{q x}^{x} f^{p-1}(g(t)) f^{\prime}(g(t)) g^{\prime}(t) \mathrm{d} t \\
& \leq \frac{p f^{p-1}(g(x)) f^{\prime}(g(x))}{(1-q) x} \int_{q x}^{x} g^{\prime}(t) \mathrm{d} t \\
& =\frac{p f^{p-1}(g(x)) f^{\prime}(g(x))}{(1-q) x}(g(x)-g(q x)) \\
& =p f^{p-1}(g(x)) f^{\prime}(g(x)) D_{q} g(x) .
\end{aligned}
$$

Also,

$$
\begin{aligned}
D_{q} f^{p}(g(x)) & \geq \frac{p f^{p-1}(g(q x)) f^{\prime}(g(q x))}{(1-q) x} \int_{q x}^{x} g^{\prime}(t) \mathrm{d} t \\
& =p f^{p-1}(g(q x)) f^{\prime}(g(q x)) D_{q} g(x) .
\end{aligned}
$$

Lemma 2.2. Define

$$
D_{q}(f, g)=\frac{f(g(x))-f(g(q x))}{g(x)-g(q x)} .
$$

Then

$$
D_{q} f(g(x))=D_{q}(f, g) D_{q} g(x) .
$$

Proof. It is follows as

$$
\begin{aligned}
& D_{q} f(g(x))=\frac{f(g(x))-f(g(q x))}{(1-q) x} \\
& =\frac{f(g(x))-f(g(q x))}{g(x)-g(q x)} \frac{g(x)-g(q x)}{(1-q) x} \\
& =D_{q}(f, g) D_{q} g(x) .
\end{aligned}
$$

Theorem 2.3. Let $f(x), f^{\prime}(x), g(x)$ be nonnegative, nondecreasing functions defined on $[a, b]_{q}$ satisfies

$$
\begin{aligned}
& (\alpha-\gamma) f^{\alpha-\gamma-1}(g(q x)) f^{\prime}(g(q x)) D_{q} g(x) \\
& \geq \beta f^{\gamma(\beta-1)}(g(x))(x-a)^{\beta-1} D_{q}^{2} g(x) \\
& +\beta(\beta-1) f^{\gamma(\beta-1)}(g(x))(x-a)^{\beta-2} D_{q} g(x)
\end{aligned}
$$

for $\alpha \geq \gamma+1, \quad \beta \geq 2$, then

$$
\int_{a}^{b} f^{\alpha}(g(x)) \mathrm{d}_{q} x \geq\left(\int_{a}^{b} f^{\gamma}(g(x)) \mathrm{d}_{q} x\right)^{\beta} .
$$

Proof. Let

$$
M(x)=\int_{a}^{x} f^{\alpha}(g(t)) \mathrm{d}_{q} t-\left(\int_{a}^{x} f^{\gamma}(g(t)) \mathrm{d}_{q} t\right)^{\beta},
$$

and

$$
h(g(x))=\int_{a}^{x} f^{\gamma}(g(t)) \mathrm{d}_{q} t .
$$

Then via Lemma 2.1, we have

$$
\begin{aligned}
& D_{q} M(x)=f^{\alpha}(g(x))-D_{q} h^{\beta}(g(x)) \\
& \geq f^{\alpha}(g(x))-\beta h^{\beta-1}(g(x)) h^{\prime}(g(x)) D_{q} g(x) \\
& =f^{\alpha}(g(x))-\beta h^{\beta-1}(g(x)) f^{\gamma}(g(x)) D_{q} g(x) \\
& =f^{\gamma}(g(x))\left(f^{\alpha-\gamma}(g(x))-\beta h^{\beta-1}(g(x)) D_{q} g(x)\right) \\
& :=f^{\gamma}(g(x)) F(g(x)) .
\end{aligned}
$$

As $h(g(x)) \leq f^{\gamma}(g(x))(x-a)$, then 


$$
\begin{aligned}
& D_{q} f(g(x))=D_{q} f^{\alpha-\gamma}(g(x))-\beta D_{q}\left(h^{\beta-1}(g(x)) D_{q} g(x)\right) \\
&=D_{q} f^{\alpha-\gamma}(g(x))-\beta h^{\beta-1}(g(x)) D_{q}^{2} g(x)-\beta D_{q} h^{\beta-1}(g(x)) D_{q} g(q x) \\
& \geq(\alpha-\gamma) f^{\alpha-\gamma-1}(g(q x)) f^{\prime}(g(q x)) D_{q} g(x)-\beta h^{\beta-1}(g(x)) D_{q}^{2} g(x) \\
&-\beta(\beta-1) h^{\beta-2}(g(x)) h^{\prime}(g(x)) D_{q} g(x) \\
& \geq(\alpha-\gamma) f^{\alpha-\gamma-1}(g(q x)) f^{\prime}(g(q x)) D_{q} g(x)-\beta h^{\beta-1}(g(x)) D_{q}^{2} g(x) \\
&-\beta(\beta-1) h^{\beta-2}(g(x)) h^{\gamma}(g(x)) D_{q} g(x) \\
& \geq(\alpha-\gamma) f^{\alpha-\gamma-1}(g(q x)) f^{\prime}(g(q x)) D_{q} g(x)-\beta f^{\gamma(\beta-1)}(g(x))(x-a)^{\beta-1} D_{q}^{2} g(x) \\
&-\beta(\beta-1) f^{\gamma(\beta-1)}(g(x))(x-a)^{\beta-2} D_{q} g(x)
\end{aligned}
$$

The above implies $D_{q} F(g(x)) \geq 0$, by (2.4), which implies $F(g(x)) \geq 0$ and hence $D_{q} M(x) \geq 0$, so that $M(x) \geq 0$.

Theorem 2.4. Let $f(x), f^{\prime}(x), g(x)$ be nonnegative, nondecreasing functions defined on $\left[b q^{n+m}, b\right]_{q}$ satisfies

$$
\begin{aligned}
& (\alpha-\gamma) f^{\alpha-\gamma-1}(g(q x)) f^{\prime}(g(q x)) D_{q} g(x) \\
& \geq \beta f^{\gamma(\beta-1)}\left(g\left(q^{m} x\right)\right)(x-a)^{\beta-1} D_{q}^{2} g(x) \\
& +\beta(\beta-1) f^{\gamma(\beta-1)}\left(g\left(q^{m} x\right)\right)(x-a)^{\beta-2} D_{q} g(x),
\end{aligned}
$$

for $\alpha \geq \gamma+1, \beta \geq 2$, then

$$
\int_{a}^{b} f^{\alpha}(g(x)) \mathrm{d}_{q} x \geq\left(\int_{a}^{b} f^{\gamma}\left(g\left(q^{m} t\right)\right) \mathrm{d}_{q} t\right)^{\beta}
$$

Proof. Let

$$
N(x)=\int_{a}^{x} f^{\alpha}(g(t)) \mathrm{d}_{q} t-\left(\int_{a}^{x} f^{\gamma}\left(g\left(q^{m} t\right)\right) \mathrm{d}_{q} t\right)^{\beta},
$$

and

$$
h(g(x))=\int_{a}^{x} f^{\gamma}\left(g\left(q^{m} t\right)\right) \mathrm{d}_{q} t .
$$

Then via Lemma 2.1, we have

$$
\begin{aligned}
& D_{q} N(x)=f^{\alpha}(g(x))-D_{q} h^{\beta}(g(x)) \geq f^{\alpha}(g(x))-\beta h^{\beta-1}(g(x)) h^{\prime}(g(x)) D_{q} g(x) \\
& =f^{\alpha}(g(x))-\beta h^{\beta-1}(g(x)) f^{\gamma}\left(g\left(q^{m} x\right)\right) D_{q} g(x) \geq f^{\alpha}(g(x))-\beta h^{\beta-1}(g(x)) f^{\gamma}(g(x)) D_{q} g(x) \\
& =f^{\gamma}(g(x))\left(f^{\alpha-\gamma}(g(x))-\beta h^{\beta-1}(g(x)) D_{q} g(x)\right):=f(g(x)) F(g(x)) .
\end{aligned}
$$

As $h(g(x)) \leq f^{\gamma}\left(g\left(q^{m} x\right)\right)(x-a)$, then

$$
\begin{array}{rl}
D_{q} & f(g(x))=D_{q} f^{\alpha-\gamma}(g(x))-\beta D_{q}\left(h^{\beta-1}(g(x)) D_{q} g(x)\right) \\
= & D_{q} f^{\alpha-\gamma}(g(x))-\beta h^{\beta-1}(g(x)) D_{q}^{2} g(x)-\beta D_{q} h^{\beta-1}(g(x)) D_{q} g(q x) \\
\geq & (\alpha-\gamma) f^{\alpha-\gamma-1}(g(q x)) f^{\prime}(g(q x)) D_{q} g(x)-\beta h^{\beta-1}(g(x)) D_{q}^{2} g(x) \\
& -\beta(\beta-1) h^{\beta-2}(g(x)) h^{\prime}(g(x)) D_{q} g(x) \\
\geq & (\alpha-\gamma) f^{\alpha-\gamma-1}(g(q x)) f^{\prime}(g(q x)) D_{q} g(x)-\beta h^{\beta-1}(g(x)) D_{q}^{2} g(x) \\
& -\beta(\beta-1) h^{\beta-2}(g(x)) h^{\gamma}\left(g\left(q^{m} x\right)\right) D_{q} g(x) \\
\geq & (\alpha-\gamma) f^{\alpha-\gamma-1}(g(q x)) f^{\prime}(g(q x)) D_{q} g(x)-\beta f^{\gamma(\beta-1)}\left(g\left(q^{m} x\right)\right)(x-a)^{\beta-1} D_{q}^{2} g(x) \\
& -\beta(\beta-1) f^{\gamma(\beta-1)}\left(g\left(q^{m} x\right)\right)(x-a)^{\beta-2} D_{q} g(x)
\end{array}
$$

The above implies $D_{q} F(g(x)) \geq 0$, by (2.4), which implies $F(g(x)) \geq 0$ and hence $D_{q} N(x) \geq 0$, so that $N(x) \geq 0$.

Theorem 2.5. Let $f, g, h$ be nonnegative functions, $h$ defined on $[a, b]_{q}$ and $f, g$ are defined on $R(h), g$, $h$ are nondecreasing with $g(a)=h(a)=0$. If

$$
\int_{x}^{b} f^{\beta}(h(t)) \mathrm{d}_{q} t \geq \int_{s}^{b} g^{\beta}(h(t)) \mathrm{d}_{q} t, \quad \forall t \in[a, b]_{q},
$$

then

$$
\int_{a}^{b} f^{\alpha+\beta}(h(x)) \mathrm{d}_{q} x \geq \int_{a}^{b} f^{\beta}(h(x)) g^{\alpha}(h(x)) \mathrm{d}_{q} x
$$


for all $\alpha, \beta>0$.

Proof. Since by Lemma 2.2,

$g^{\alpha}(h(x))=\int_{a}^{x} D_{q} g^{\alpha}(h(t)) \mathrm{d}_{q} t=\int_{a}^{x} D_{q}\left(g^{\alpha}, h\right) D_{q} h(t) \mathrm{d}_{q} t$,

then, we have

$$
\begin{aligned}
& \int_{a}^{b} f^{\beta}(h(x)) g^{\alpha}(h(x)) \mathrm{d}_{q} x \\
& =\int_{a}^{b} f^{\beta}(h(x)) \int_{a}^{x} D_{q}\left(g^{\alpha}, h\right) D_{q} h(t) \mathrm{d}_{q} t \mathrm{~d}_{q} x \\
& =\int_{a}^{b} D_{q}\left(g^{\alpha}, h\right) D_{q} h(t)\left(\int_{t}^{b} f^{\beta}(h(x)) \mathrm{d}_{q} x\right) \mathrm{d}_{q} t \\
& \geq \int_{a}^{b} D_{q}\left(g^{\alpha}, h\right) D_{q} h(t)\left(\int_{t}^{b} g^{\beta}(h(x)) \mathrm{d}_{q} x\right) \mathrm{d}_{q} t \\
& =\int_{a}^{b} g^{\beta}(h(x)) \mathrm{d}_{q} x\left(\int_{a}^{x} D_{q}\left(g^{\alpha}, h\right) D_{q} h(t) \mathrm{d}_{q} t\right) \mathrm{d}_{q} x \\
& =\int_{a}^{b} g^{\alpha+\beta}(h(x)) \mathrm{d}_{q} x .
\end{aligned}
$$

Using the arithmetic-geometric inequality yields

$$
\begin{aligned}
& \frac{\beta}{\alpha+\beta} f^{\alpha+\beta}(h(x))+\frac{\alpha}{\alpha+\beta} g^{\alpha+\beta}(h(x)) \\
& \geq f^{\beta}(h(x)) g^{\alpha}(h(x)) .
\end{aligned}
$$

Integrating the above inequality yields

$$
\begin{aligned}
& \frac{\beta}{\alpha+\beta} \int_{a}^{b} f^{\alpha+\beta}(h(x)) \mathrm{d}_{q} x+\frac{\alpha}{\alpha+\beta} \int_{a}^{b} g^{\alpha+\beta}(h(x)) \mathrm{d}_{q} x \\
& \geq \int_{a}^{b} f^{\beta}(h(x)) g^{\alpha}(h(x)) \mathrm{d}_{q} x,
\end{aligned}
$$

which implies via (2.9),

$$
\begin{aligned}
& \int_{a}^{b} f^{\beta}(h(x)) g^{\alpha}(h(x)) \mathrm{d}_{q} x \leq \frac{\beta}{\alpha+\beta} \int_{a}^{b} f^{\alpha+\beta}(h(x)) \mathrm{d}_{q} x \\
& +\frac{\alpha}{\alpha+\beta} \int_{a}^{b} f^{\beta}(h(x)) g^{\alpha}(h(x)) \mathrm{d}_{q} x .
\end{aligned}
$$

and hence

$$
\int_{a}^{b} f^{\alpha+\beta}(h(x)) \mathrm{d}_{q} x \geq \int_{a}^{b} f^{\beta}(h(x)) g^{\alpha}(h(x)) \mathrm{d}_{q} x .
$$

Theorem 2.6. Let $f, g, h$ be nonnegative functions, $h$ defined on $[a, b]_{q}$ and $f, g$ are defined on $R(h)$, $g, h$ are nondecreasing with $g(a)=h(a)=0$. If

$$
\int_{x}^{b} f(h(t)) \mathrm{d}_{q} t \geq \int_{s}^{b} g(h(t)) \mathrm{d}_{q} t, \forall t \in[a, b]_{q},
$$

then

$$
\int_{a}^{b} f^{\alpha+\beta}(h(x)) \mathrm{d}_{q} x \geq \int_{a}^{b} f^{\beta}(h(x)) g^{\alpha}(h(x)) \mathrm{d}_{q} x
$$

for all $\alpha \geq 1, \beta>0$.

Proof. For $\alpha \geq 1$, we have

$$
\begin{aligned}
& \int_{a}^{b} f(h(x)) g^{\alpha-1}(h(x)) \mathrm{d}_{q} x \\
& =\int_{a}^{b} f(h(x)) \int_{a}^{x} D_{q}\left(g^{\alpha-1}, h\right) D_{q}(h(t)) \mathrm{d}_{q} t \mathrm{~d}_{q} x \\
& =\int_{a}^{b} D_{q}\left(g^{\alpha-1}, h\right) D_{q}(h(t))\left(\int_{t}^{b} f(h(x)) \mathrm{d}_{q} x\right) \mathrm{d}_{q} t \\
& \geq \int_{a}^{b} D_{q}\left(g^{\alpha-1}, h\right) D_{q}(h(t))\left(\int_{t}^{b} g(h(x)) \mathrm{d}_{q} x\right) \mathrm{d}_{q} t \\
& =\int_{a}^{b} g(h(x)) \int_{a}^{x} D_{q}\left(g^{\alpha-1}, h\right) D_{q}(h(t)) \mathrm{d}_{q} t \mathrm{~d}_{q} x \\
& =\int_{a}^{b} g^{\alpha}(h(x)) \mathrm{d}_{q} x .
\end{aligned}
$$

Now, applying the AG inequality, we have for $\alpha \geq 1$, $\frac{1}{\alpha} f^{\alpha}(h(x))+\frac{\alpha-1}{\alpha} g^{\alpha}(h(x)) \geq f(h(x)) g^{\alpha-1}(h(x))$.

On integrating the above inequality with the using of (2.11) leads to

$$
\begin{aligned}
& \int_{a}^{b} g^{\alpha}(h(x)) \mathrm{d}_{q} x \leq \int_{a}^{b} f(h(x)) g^{\alpha-1}(h(x)) \mathrm{d} x_{q} \\
& \leq \frac{1}{\alpha} \int_{a}^{b} g^{\alpha}(h(x)) \mathrm{d}_{q} x+\frac{\alpha-1}{\alpha} \int_{a}^{b} g^{\alpha}(h(x)) \mathrm{d}_{q} x,
\end{aligned}
$$

which implies

$$
\int_{a}^{b} g^{\alpha}(h(x)) \mathrm{d}_{q} x \leq \int_{a}^{b} f^{\alpha}(h(x)) \mathrm{d}_{q} x .
$$

Again, by the AG inequality, and via integration

$$
\begin{aligned}
& \int_{a}^{b} f^{\alpha}(h(x)) g^{\beta}(h(x)) \mathrm{d}_{q} x \leq \frac{\alpha}{\alpha+\beta} \int_{a}^{b} f^{\alpha+\beta}(h(x)) \mathrm{d}_{q} x \\
& +\frac{\beta}{\alpha+\beta} \int_{a}^{b} g^{\alpha+\beta}(h(x)) \mathrm{d}_{q} x,
\end{aligned}
$$

and the above implies, by (2.12),

$$
\int_{a}^{b} f^{\alpha}(h(x)) g^{\beta}(h(x)) \mathrm{d}_{q} x \leq \int_{a}^{b} f^{\alpha+\beta}(h(x)) \mathrm{d}_{q} x .
$$

The other way round direction may be obtained from the following.

Theorem 2.7. Let $f, g, h$ be nonnegative functions, $h$ defined on $[a, b]_{q}$ and $f, g$ are defined on $R(h), g, h$ are nondecreasing with $g(a)=h(a)=0$. If 
$\int_{x}^{b} f^{\beta}(h(t)) \mathrm{d}_{q} t \geq \int_{s}^{b} g^{\beta}(h(t)) \mathrm{d}_{q} t, \quad \forall t \in[a, b]_{q}$,

then

$$
\int_{a}^{b} f^{\beta-\alpha}(h(x)) \mathrm{d}_{q} x \geq \int_{a}^{b} f^{\beta}(h(x)) g^{-\alpha}(h(x)) \mathrm{d}_{q} x
$$

for all $\beta>\alpha>0$.

Proof. We have, via Lemma 2.2,

$$
\begin{aligned}
& \int_{a}^{b} f^{\beta}(h(x)) g^{-\alpha}(h(x)) \mathrm{d}_{q} x \\
& =\int_{a}^{b} f^{\beta}(h(x)) \int_{a}^{x} D_{q}\left(g^{-\alpha}, h\right) D_{q} h(t) \mathrm{d}_{q} t \mathrm{~d}_{q} x \\
& =\int_{a}^{b} D_{q}\left(g^{-\alpha}, h\right) D_{q} h(t)\left(\int_{t}^{b} f^{\beta}(h(x)) \mathrm{d}_{q} x\right) \mathrm{d}_{q} t \\
& \geq \int_{a}^{b} D_{q}\left(g^{-\alpha}, h\right) D_{q} h(t)\left(\int_{t}^{b} g^{\beta}(h(x)) \mathrm{d}_{q} x\right) \mathrm{d}_{q} t \\
& =\int_{a}^{b} g^{\beta}(h(x)) \mathrm{d}_{q} x\left(\int_{a}^{x} D_{q}\left(g^{-\alpha}, h\right) D_{q} h(t) \mathrm{d}_{q} t\right) \mathrm{d}_{q} x \\
& =\int_{a}^{b} g^{\beta-\alpha}(h(x)) \mathrm{d}_{q} x .
\end{aligned}
$$

Making use of the AG inequality,

$$
\begin{aligned}
& \frac{\beta}{\beta-\alpha} f^{\beta-\alpha}(h(x))-\frac{\alpha}{\beta-\alpha} g^{\beta-\alpha}(h(x)) \\
& \leq f^{\beta}(h(x)) g^{-\alpha}(h(x)) .
\end{aligned}
$$

Integrating the above inequality, with making use of (2.14) yield

$$
\begin{aligned}
& \frac{\beta}{\beta-\alpha} \int_{a}^{b} f^{\beta-\alpha}(h(x)) \mathrm{d}_{q} x \leq \frac{\alpha}{\beta-\alpha} \int_{a}^{b} g^{\beta-\alpha}(h(x)) \mathrm{d}_{q} x \\
& \quad+\int_{a}^{b} f^{\beta}(x) g^{-\alpha}(h(x)) \mathrm{d}_{q} x \\
& \leq \frac{\alpha}{\beta-\alpha} \int_{a}^{b} f^{\beta}(x) g^{-\alpha}(h(x)) \mathrm{d}_{q} x \\
& \quad+\int_{a}^{b} f^{\beta}(x) g^{-\alpha}(h(x)) \mathrm{d}_{q} x \\
& =\frac{\beta}{\beta-\alpha} \int_{a}^{b} f^{\beta}(x) g^{-\alpha}(h(x)) \mathrm{d}_{q} x .
\end{aligned}
$$

\section{References}

[1] Y. Miao and F. Qi, "Several q-Integral Inequalities," Journal of Mathematical Inequalities, Vol. 3, No. 1, 2009, pp. 115-121.

[2] K. Brahim, N. Bettaibi and M. Sellemi, "On Some Feng Qi Type q-Intagral Inequlities,” Pure Applied Mathematics, Vol. 9, No. 2, 2008, Art. 43.

[3] E. W. Weisstein, “q-Derivative,” Math World-A Wolfram Web Resource,” 2010. http://mathword. Wolfram .com/q-Derivative.html

[4] E. W. Weisstein, “q-Integral,” Math World-A Wolfram Web Resource,” 2010.

http://mathword. Wolfram .com/q-integral.html

[5] F. H. Jackson, “On q-Definite Integrals,” Pure Applied Mathematics, Vol. 41, No. 15, 1910, pp. 193-203.

[6] V. Kac and P. Cheung, “Quantum Calculus,” Universitext, Springer-Verlag, New York, 2003. 